\title{
Narrowing of $d$ bands of FeCo layers intercalated under graphene
}

Cite as: Appl. Phys. Lett. 118, 121602 (2021); https://doi.org/10.1063/5.0047266

Submitted: 11 February 2021. Accepted: 08 March 2021. Published Online: 22 March 2021

Daniela Pacilè, (ID Claudia Cardoso, (iD) Giulia Avvisati, (iD) Ivana Vobornik, (iD) Carlo Mariani, (iD) Dario A. Leon, (iD) Pietro Bonfà, (D) Daniele Varsano, (D) Andrea Ferretti, and (D) Maria Grazia Betti

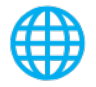

Challenge us.

What are your needs for periodic signal detection?

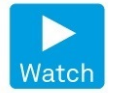

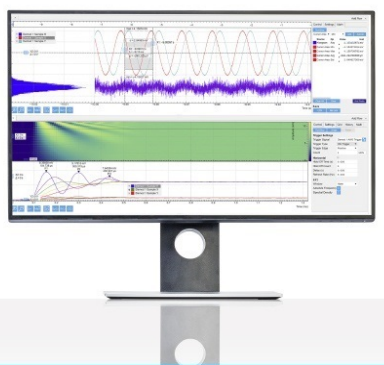

- Zurich

Instruments 


\title{
Narrowing of $d$ bands of FeCo layers intercalated under graphene
}

\author{
Cite as: Appl. Phys. Lett. 118, 121602 (2021); doi: 10.1063/5.0047266 \\ Submitted: 11 February 2021 - Accepted: 8 March 2021 . \\ Published Online: 22 March 2021

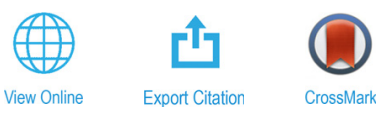
Daniela Pacilè,, ${ }^{1, a)}$ Claudia Cardoso, ${ }^{2, a)}$ (iD Giulia Avvisati, ${ }^{3}$ (D) Ivana Vobornik, ${ }^{4}$ (iD Carlo Mariani, ${ }^{3}$ (D) Dario A. Leon, ${ }^{2,5}$ Pietro Bonfà, 2,6 (D) Daniele Varsano, ${ }^{2}$ (iD Andrea Ferretti, ${ }^{2}$ (D) and Maria Grazia Betti ${ }^{3}$ (D)

\author{
AFFILIATIONS \\ 'Dipartimento di Fisica, Università della Calabria, I-87036 Arcavacata di Rende (Cs), Italy \\ ${ }^{2}$ Centro S3, CNR-Istituto Nanoscienze, I-41125 Modena, Italy \\ ${ }^{3}$ Dipartimento di Fisica, Università di Roma “La Sapienza," I-00185 Roma, Italy \\ ${ }^{4}$ Istituto Officina dei Materiali (IOM)-CNR, Laboratorio TASC, Area Science Park, S.S.14, Km 163.5, 34149 Trieste, Italy \\ ${ }^{5}$ Università di Modena e Reggio Emilia, I-41125 Modena, Italy \\ ${ }^{6}$ Università di Parma, Dipartimento di Scienze Matematiche, Fisiche e Informatiche, I-43124 Parma, Italy
}

a) Authors to whom correspondence should to addressed: daniela.pacile@fis.unical.it and claudiamaria.cardosopereira@nano.cnr.it

\begin{abstract}
We report on the electronic properties of an artificial system obtained by the intercalation of equiatomic FeCo layers under graphene grown on $\operatorname{Ir}(111)$. Upon intercalation, the FeCo film grows epitaxially on $\operatorname{Ir}(111)$, resulting in a lattice-mismatched system. By performing density functional theory calculations, we show that the intercalated FeCo layer leads to a pronounced corrugation of the graphene film. At the same time, the FeCo intercalated layers induce a clear transition from a nearly undisturbed to a strongly hybridized graphene $\pi$-band, as measured by angle-resolved photoemission spectroscopy. A comparison of experimental results with the computed band structure and the projected density of states unveils a spin-selective hybridization between the $\pi$ band of graphene and FeCo-3d states. Our results demonstrate that the reduced dimensionality, as well as the hybridization within the FeCo layers, induces a narrowing and a clear splitting of Fe $3 d-$ up and Fe $3 d$-down-spin bands of the confined FeCo layers with respect to bulk Fe and Co.
\end{abstract}

Published under license by AIP Publishing. https://doi.org/10.1063/5.0047266

Ferromagnetic metals (FMs) and their alloys can be finely manipulated by changing their chemical composition ${ }^{1}$ and structural configuration, ${ }^{2}$ and by reducing the dimensionality. ${ }^{3,4}$ Modified symmetry and scaled dimension, indeed, may induce in FMs higher magnetic moments and larger uniaxial magnetic anisotropy energy (MAE) with respect to their 3D counterparts. ${ }^{4-8}$ The enhanced magnetism in nanostructures can be used, e.g., for engineering spintronic devices, high-density magnetic storage, ${ }^{10}$ and permanent magnets. ${ }^{11}$

Among all iron-based alloys, including also pure iron, equiatomic FeCo exhibits the highest Curie temperature and magnetic moment driven by an almost filled Fe majority spin band. ${ }^{12-15}$ On the other hand, due to its cubic symmetry, the FeCo alloy also shows a low MAE. ${ }^{16}$ Indeed, modifying dimensionality or symmetry has been shown to be a viable route in order to improve the magnetic response of FeCo alloys, ${ }^{8,17}$ although clustering and granularity may hinder a controlled growth of artificial systems. ${ }^{18}$ Intercalation of metals underneath graphene $(\mathrm{Gr})$ has led to the formation of atomically smooth metallic layers, ${ }^{19-29}$ where $\mathrm{Gr}$ on top is also a protective membrane against contaminants. ${ }^{30,31}$ Although several metals have been intercalated under $\mathrm{Gr}$, the combined intercalation of Fe and Co to form an equiatomic alloy is more challenging, as segregation without intermixing may be dominant. ${ }^{31}$ Recently, we have grown homogeneous and smooth $\mathrm{Fe}_{x} \mathrm{Co}_{1-x}$ layers underneath $\mathrm{Gr} / \mathrm{Ir}(111)$, co-depositing $\mathrm{Fe}$ and $\mathrm{Co}$ at the same evaporation rate and keeping the substrate temperature at about $500 \mathrm{~K}$, to avoid any $\mathrm{Fe}-\mathrm{Ir}$ and Co-Ir alloying formation and clustering. By performing X-ray Magnetic Circular Dichroism (XMCD) measurements, we showed that Fe and Co are well intermixed and magnetically coupled underneath $\mathrm{Gr}$, leading to an enhancement of the magnetic moments with respect to pure $\mathrm{Fe}$ and Co films and exhibiting a strong ferromagnetic exchange coupling between the two species.

In the present work, we investigate the electronic and structural properties of the equiatomic FeCo layers (about 1-2 monolayers of thickness) grown epitaxially on $\operatorname{Ir}(111)$ underneath a Gr cover, by means of Angular Resolved Photoemission Spectroscopy (ARPES) and Density Functional Theory (DFT). We show that the reduced 
dimensionality in the artificially confined system leads to a narrowing and a redistribution of majority and minority $3 d$ states with respect to the pure species, also enhancing the splitting between $\mathrm{Fe} 3 d$-up and $\mathrm{Fe}$ $3 d$-down-spin bands. Our description of the electronic structure in equiatomic FeCo layers sheds light on the enhanced magnetism previously demonstrated by $\mathrm{XMCD}^{8}$ and provides a useful insight into the engineering of low dimensional FM alloys.

The $\operatorname{Ir}(111)$ surface was cleaned by cycles of sputtering $\left(\mathrm{Ar}^{+}\right.$, $1.5-2.0 \mathrm{keV}$ ) and annealing at about $1300 \mathrm{~K}$. The Gr sheet is obtained by exposing the clean surface to $5 \times 10^{-8}-2 \times 10^{-7}$ mbar of $\mathrm{C}_{2} \mathrm{H}_{4}$ and annealing at $1300-1320 \mathrm{~K}$. Metallic $\mathrm{Fe}$ and Co were simultaneously deposited, at previously calibrated same evaporation rates (about $0.3 \AA / \mathrm{min}$ ) to deposit nominal 3 monolayers (MLs) of FeCo on the $\mathrm{Gr} / \mathrm{Ir}(111)$ substrate kept in the range of $500-530 \mathrm{~K}$. As recently reported, ${ }^{8}$ core level photoemission and absorption spectroscopy unveil homogeneous intercalation of equiatomic FeCo layers, without any alloy formation with the underlying substrate ${ }^{8}$ (as shown in the supplementary material), at variance e.g., with recent observations for Mn intercalation on $\mathrm{Gr} / \mathrm{Ir}$.

The sample preparation and ARPES experiments were carried out at the APE beamline of the Elettra Synchrotron Light Source (Trieste, Italy). The photoelectrons, excited with a photon energy of $86 \mathrm{eV}$, were collected with a VG Scienta DA30 electron energy analyzer, which operates in deflection mode and allows for detailed k-space mapping at fixed sample geometry.

Density functional theory (DFT) simulations were carried out using the plane wave implementation of the QUANTUM ESPRESSO ${ }^{33,34}$ distribution. We used the local density approximation (LDA) for the exchange-correlation potential, according to the Perdew-Zunger parametrization. ${ }^{35}$ Since LDA is known to underestimate the values of the orbital magnetic moments in transition metals, ${ }^{36}$ we have adopted a DFT $+\mathrm{U}$ scheme, ${ }^{37}$ with a Hubbard $\mathrm{U}$ parameter of $2 \mathrm{eV}$ for Fe and $4 \mathrm{eV}$ for Co. The choice of the values is explained in detail in the SI. Similar to our previous works, ${ }^{27,38,39}$ we simulated the Gr/1ML-FeCo/ $\operatorname{Ir}(111)$ interface considering the complete moiré induced periodicity by using a $9 \times 9$ supercell of $\operatorname{Ir}(111)$, corresponding to a $10 \times 10$ supercell of pristine Gr. More details about the structure can be found in the supplementary material.

In order to obtain a simpler representation of the $\mathrm{Gr} / \mathrm{FeCo} /$ $\operatorname{Ir}(111)$ band structure, we have applied an unfolding procedure ${ }^{40,41}$ in which the band structure computed for the $10 \times 10$ supercell is mapped into the Gr $1 \times 1$ Brillouin Zone (BZ) by using the unfold-x code. $^{41}$ This approach has been further extended to include atomic projections on Lödwin orthogonalized atomic orbitals as provided by the QUANTUM ESPRESSO package. ${ }^{33,34}$

Figure 1 shows the low energy electron diffraction (LEED) patterns of $\mathrm{Gr} / \operatorname{Ir}(111)$, taken with a primary energy of about $90 \mathrm{eV}$, before (a) and after (b) the intercalation of nominal 3ML of FeCo. The moiré superstructure characteristic of the corrugated $\mathrm{Gr} /$ $\operatorname{Ir}(111)$ system is maintained after FeCo intercalation, attesting that the equiatomic FeCo layers arrange pseudomorphically on the hexagonal $\operatorname{Ir}(111)$ surface. The moire pattern is only slightly smeared out at an increasing amount of intercalated atoms, with an intensity reduction of the extra spots. The persistence of multiple reflection spots suggests that the thickness of the intercalated FeCo layer is within 1 and 2 MLs, as deduced by comparison with the LEED patterns of pure $\mathrm{Co}^{23}$ and $\mathrm{Fe}^{27}$ intercalated systems.

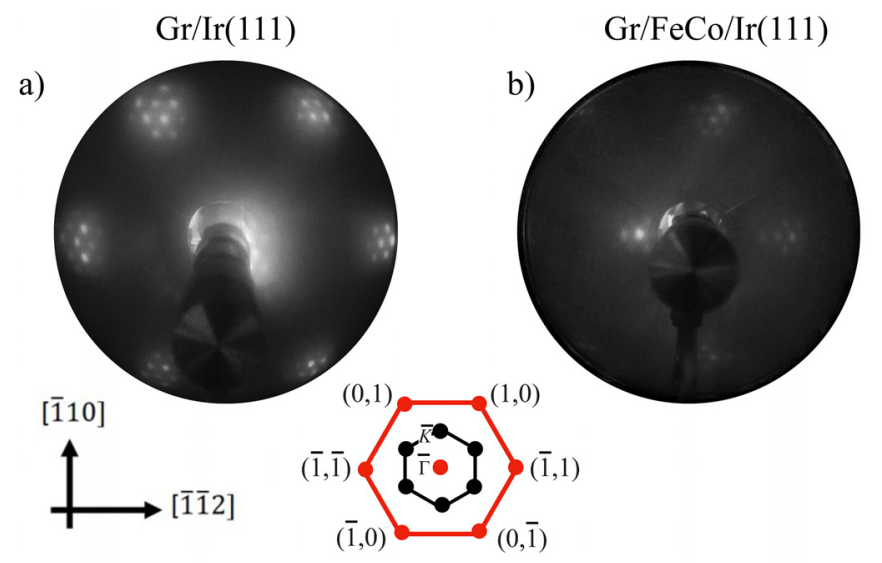

c)
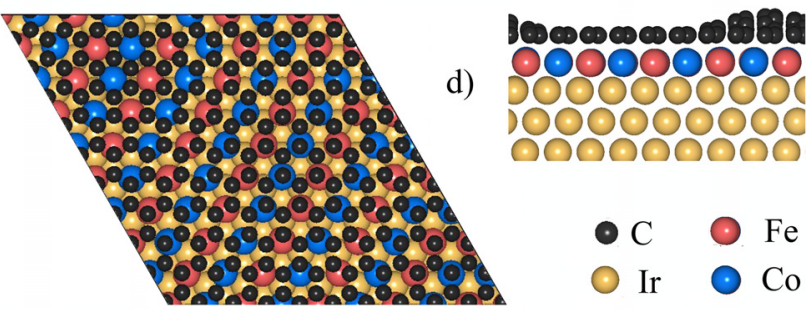

FIG. 1. Low energy electron diffraction (LEED) patterns (electron beam energy: $90 \mathrm{eV}$ ) of (a) $\mathrm{Gr} / \mathrm{Ir}(111)$ and (b) $\mathrm{Gr} / \mathrm{FeCo} / \mathrm{r}(111)$, taken slightly off-normal. In the middle: sketch of the diffraction pattern and Brillouin Zone (BZ) of $\operatorname{Ir}(111)$, referred to (a) and (b) panels. (c) Atomic structure as deduced by DFT, top view of the moiré pattern of $\mathrm{Gr} / \mathrm{FeCo} / \mathrm{Ir}(111)$ with $\mathrm{C}$ atoms represented in gray, $\mathrm{Fe}$ in red, $\mathrm{Co}$ in blue, and Ir in cream; (d) side view of the $\mathrm{Gr} / \mathrm{FeCo} / \mathrm{r}(111)$ structure showing the $\mathrm{Gr}$ corrugation, using the same colors described in the previous panel.

The $\mathrm{Gr} / \mathrm{FeCo} / \mathrm{Ir}(111)$ interface was modeled at the DFT level as described previously. The calculations indicate a large corrugation of the Gr layer $(1.40 \AA)$, with Gr-FeCo interplanar distances of $1.90 \AA$ and $3.30 \AA$ at valleys and hills, respectively, as shown in Figs. 1(c) and $1(\mathrm{~d})$. The corrugation of the $\mathrm{Gr} / \mathrm{FeCo} / \mathrm{Ir}(111)$ and the energy landscape of the stable structural configuration are similar to those computed for the pure intercalated systems $\mathrm{Gr} / \mathrm{Fe} / \mathrm{Ir}(111)(1.3 \AA)^{27}$ and $\mathrm{Gr} / \mathrm{Co} / \operatorname{Ir}(111)(1.4 \AA) .{ }^{22,38}$ It is worth noticing that we do not include van der Waals interactions in our approach since LDA has shown to give a good description of graphene corrugation and distance for $\mathrm{Gr} / \mathrm{Fe} / \mathrm{Ir}(111),{ }^{27}$ providing essentially the same scenario as DFT calculations done at the PBE level including van der Waals interactions, ${ }^{42}$ which only find slightly larger Gr-Fe distances.

To shed light on the interaction mechanism between Gr and the FeCo interface, we show angular resolved photoemission measurements and compare them with the band structure computed using Kohn-Sham DFT. Figure 2(a) shows ARPES results of $\mathrm{Gr} / \mathrm{FeCo} /$ $\operatorname{Ir}(111)$ taken along $\bar{\Gamma}-\overline{\mathrm{K}}$. The effect of Fe and Co states on the $\pi$ and $\sigma$ bands of $\mathrm{Gr}$ can be discussed by comparing the system under study with pure $\mathrm{Gr} / \mathrm{Co}$ and $\mathrm{Gr} / \mathrm{Fe}$ (either supported on $\operatorname{Ir}(111)$ or not), as reported in the literature. ${ }^{23,27,43-45}$ At the $\bar{\Gamma}$-point [Fig. 2(a)], the bottom of the $\pi$ band and the top of the $\sigma$ bands are shifted to about $10.2 \mathrm{eV}$ and $5.4 \mathrm{eV}$ of binding energy (BE), respectively, in agreement with previous results for pure $\mathrm{Fe}$ and $\mathrm{Co}$ intercalated systems. ${ }^{23,27}$ Moreover, ARPES data extracted along the direction perpendicular to 
a)

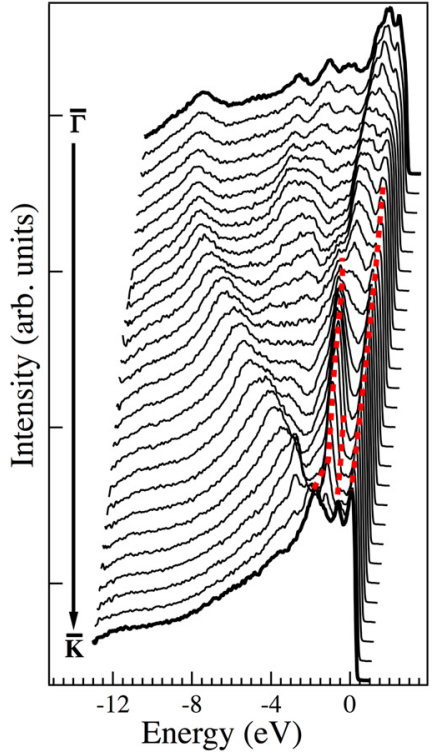

b)

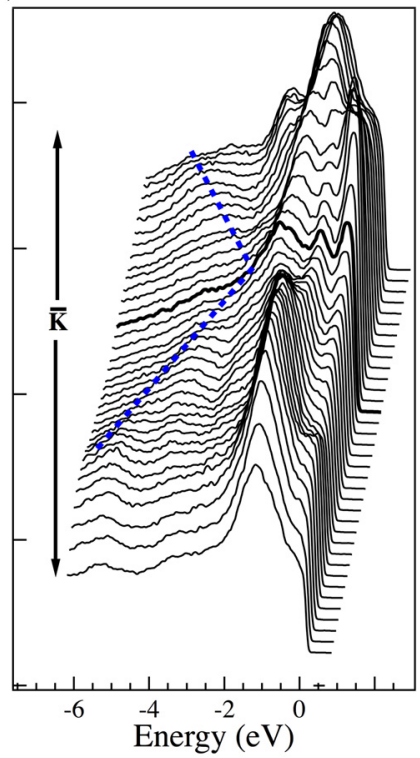

FIG. 2. (a) Selected EDCs of Gr/FeCo/lr(111), extracted from data of Fig. 3(a), acquired with a photon energy of $86 \mathrm{eV}$ and taken along the $\bar{\Gamma}-\overline{\mathrm{K}}$ direction. Red dashed lines highlight states originating from the FeCo interface. (b) Selected EDCs taken along the direction perpendicular to $\bar{\Gamma}-\bar{K}$. Blue dashed lines highlight the $\pi$ band of $\mathrm{Gr}$. The $\overline{\mathrm{K}}$-point has been extracted at an electron kinetic energy of about $80 \mathrm{eV}$.

$\bar{\Gamma}-\overline{\mathrm{K}}$ [Fig. 2(b)] show the maximum of the Gr $\pi$ band at about $2.1 \mathrm{eV}$ of BE. Other pure Gr/FM systems exhibit a $\pi$ band maximum between 2.5 and $3.0 \mathrm{eV}$, depending both on the metal and on the number of intercalated layers. ${ }^{23,27,43-46}$ The Gr/Co system, e.g., leads to a crossing point between $\pi$ bands located at $2.8 \mathrm{eV}$ of $\mathrm{BE} .^{23}$ For $\mathrm{Gr} / \mathrm{FeCo} / \mathrm{Ir}$, the lower $\mathrm{BE}$ value of the $\pi$ band maximum suggests a reduced width of the FeCo- $d$ states and thus a reduced hybridization with the $\pi$ band.

The region in the proximity of the $\overline{\mathrm{K}}$-point exhibits several features directly related to the mixed Fe-Co interface with Gr.
Specifically, in Fig. 2(a), we highlight with red dashed lines three slightly dispersing states, crossing the $\overline{\mathrm{K}}$-point at about $-1.8 \mathrm{eV}$, $-0.6 \mathrm{eV}$, and at the Fermi level $\left(E_{\mathrm{F}}\right)$. It is worth noticing that the appearance of these well-defined localized bands within $\sim 2 \mathrm{eV}$ from $E_{\mathrm{F}}$ and their dispersive behavior provide further evidence of a uniform FeCo alloy. The bands at about $4 \mathrm{eV}$ of $\mathrm{BE}$, less dispersive toward the $\overline{\mathrm{K}}$ point and resonant with the $\pi$ band of $\mathrm{Gr}$, can be attributed to the FeCo interlayer. As discussed in the following, these states are due to the majority $3 d$ states of both Co and Fe atoms, while the states close to $E_{\mathrm{F}}$ can be associated with the $\mathrm{Fe}$ and $\mathrm{Co} 3 d$ spin minority bands.

The experimental evidence of narrow bands associated with FeCo in the energy region of $E_{\mathrm{F}}$ and the hybridization of $\pi$ states of graphene is confirmed by the DFT calculations. The spin-resolved electronic structure of $\mathrm{Gr} / 1 \mathrm{ML}-\mathrm{FeCo} / \mathrm{Ir}(111)$, shown in Figs. $3(b)-3(e)$, is projected on atomic orbitals of different species and mapped into the $1 \times 1 \mathrm{Gr} \mathrm{BZ}$ along the $\bar{\Gamma}-\overline{\mathrm{K}}$ direction. With respect to free-standing $\mathrm{Gr}$, the main effect on the $\pi$ and $\sigma$ bands is a non-rigid shift toward higher BE [Fig. 3(b)], as also found in the literature for pure intercalated systems. ${ }^{23,27,43-45}$ In addition, our calculations clearly show a significant interaction of the $\mathrm{Gr} \pi$-band with the FeCo-d majority states, which is stronger than with the minority bands. This is especially evident in the wide $0-5 \mathrm{eV}$ energy range below $E_{\mathrm{F}}$ [Fig. 3(a)]. The spin-down $\pi$ band of Gr preserves its linear dispersion up to about $-2 \mathrm{eV}$, where it strongly overlaps with the FeCo minority states [Figs. 3(b)-3(d)], and their interaction pushes the $\pi$ band maximum down, in agreement with our ARPES measurements. Therefore, in line with a recent paper of $\mathrm{Gr}$ grown on a $\mathrm{Mn}_{5} \mathrm{Ge}_{3} \mathrm{FM}$ interface, the theoretical predictions demonstrate that the interaction of $\mathrm{Gr}$ with the FeCo layer gives rise to a downshifted $\mathrm{Gr} \pi$ band, which is mainly minority-spin polarized.

This picture is further confirmed and summarized by the comparison of the total band structure (central panel of Fig. 4), with the spin DOS projected on $\mathrm{C}, \mathrm{Fe}, \mathrm{Co}$, and Ir atomic orbitals (left-right panels of Fig. 4), computed within DFT for Gr/1ML-FeCo/Ir(111). From the pDOS, we can see that the Fe and Co $3 d$ states, particularly in the majority spin channel, almost coincide (see the supplementary material for a detailed analysis, including the role of DFT $+U$ corrections). The majority spin channel is almost filled, with an intense peak a) ARPES

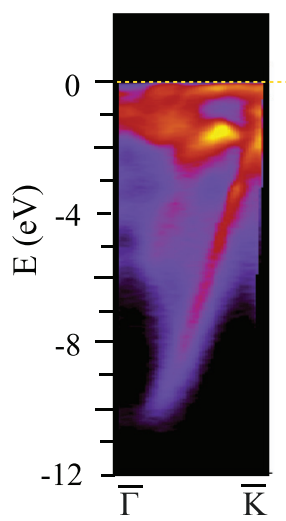

b) $\quad \mathrm{Gr}$

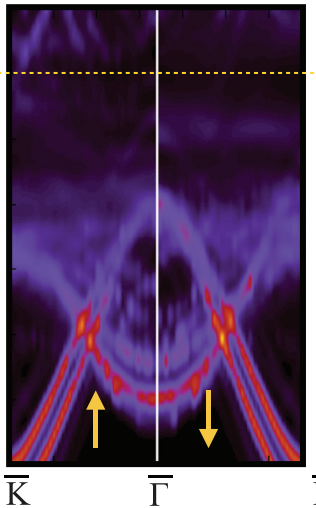

c)

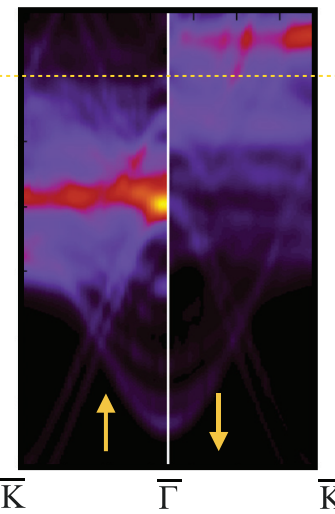

d)

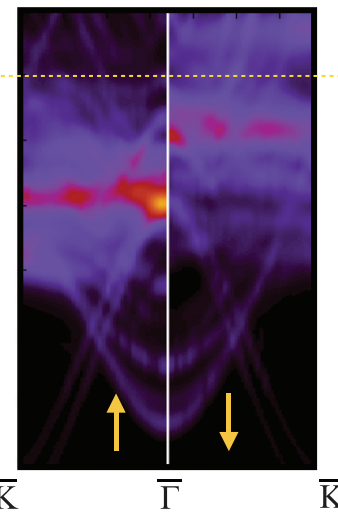

e) $\quad \operatorname{Ir}$

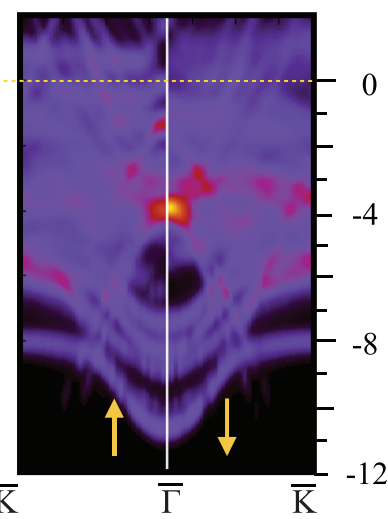

FIG. 3. Comparison between ARPES measurements of $\mathrm{Gr} / \mathrm{FeCo} / \mathrm{lr}(111)$ taken along the $\bar{\Gamma}-\overline{\mathrm{K}}$ direction (a) and the band structure computed for the $10 \times 10$ supercell of $\mathrm{Gr} /$ $1 \mathrm{ML}-\mathrm{FeCo} / \mathrm{lr}(111)$, mapped into the graphene $1 \times 1 \mathrm{BZ}$ and projected into the atomic orbitals of the different atomic species (b)-(e). 


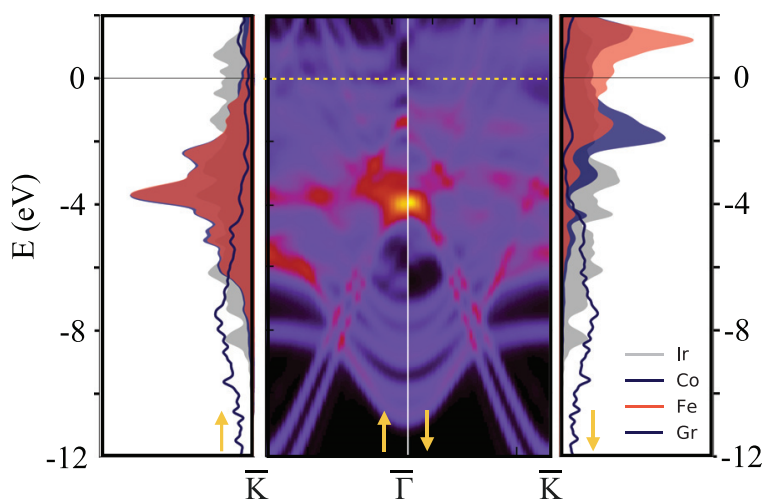

FIG. 4. Central panel: majority and minority spin band structure computed within DFT for Gr/1ML-FeCo/lr(111). Left (right) panel: majority (minority) spin DOS computed within DFT and projected on $\mathrm{C}, \mathrm{Fe}, \mathrm{Co}$, and Ir atomic orbitals.

centered at about $-4 \mathrm{eV}$, where the spin-up Dirac cone is strongly hybridized and almost quenched. In the minority spin channel, the main superimposition of $\mathrm{Fe}$ and $\mathrm{Co}$ pDOS peaks is seen at about $-2 \mathrm{eV}$ and at the Fermi level, where, indeed, the transition metal states appear in our ARPES measurements.

The density of states and the spin electronic bands for bulk FeCo alloys have been deeply investigated and discussed in the literature. $^{4,47,48}$ The strong hybridization of Fe and Co in the bulk alloy produces an increase in the Fe exchange splitting and a saturation of the magnetic moments. ${ }^{47,48}$ More specifically, it has been shown that at about $30 \%$ Fe content, the atomic magnetic moments become almost independent of the alloy composition due to a redistribution of $3 d$ minority electrons to the $3 d$ majority states at the $\mathrm{Fe}$ sites. Furthermore, the reduced dimensionality and number of nearest neighbors at the FeCo interface also influence the $d$ bandwidth and the spin splitting of the states. ${ }^{4,47,48}$

Despite a number of differences with the $\mathrm{Gr} / \mathrm{FeCo} / \mathrm{Ir}$ interfaces studied here, such as the interaction with Gr or with the Ir surface, some of the features of FeCo alloys subsist. In particular, we observe a larger spin-splitting between majority and minority spin channels and a narrowing bandwidth of the $3 d$ states for this confined FeCo layer with respect to bulk $\mathrm{Fe}$ and FeCo. ${ }^{47} \mathrm{~A}$ detailed comparison between the $\mathrm{Gr} / \mathrm{FeCo} / \mathrm{Ir}(111)$ interface and the bulk systems is presented in the SI. From the DFT simulations, the spin-resolved DOS of Fe and Co show almost completely filled $3 d$ states in the spin-up channel, with about 4.76(4.70) electrons for $\mathrm{Fe}(\mathrm{Co})$, while about 2.08(3.09) electrons are localized in the $3 d$ spin-down channel. Though the energy position of the FeCo $3 d$ states depends on the values of $U$ chosen for the Hubbard correction, there is always a significant overlap between the Fe and Co states, while the Gr states are almost insensitive to different values of $\mathrm{U}$, as described in the supplementary material.

Therefore, the results shown in Fig. 4 demonstrate that the reduced symmetry and the structural configuration of the intercalated equiatomic FeCo layer induce a clear splitting of Fe $3 d$-up and down bands, with a narrower bandwidth with respect to Fe bulk.

This picture is consistent with previous XMCD measurements on $\mathrm{Gr} / \mathrm{FeCo} / \mathrm{Ir}(111)$, showing a sensible enhancement of the magnetic moments ${ }^{8}$ with a strong ferromagnetic coupling between $\mathrm{Fe}$ and Co.
In conclusion, within the present study, we characterize the electronic and structural properties of few FeCo layers, embedded between Gr and $\operatorname{Ir}(111)$. By means of ARPES, we show that the interaction of the $\pi$ band of Gr with FeCo- $3 d$ states pushes the strongly hybridized Dirac cone toward higher BE in analogy with pure intercalated systems. However, in proximity of $E_{\mathrm{F}}$, we identify dispersive states associated with the FeCo alloy. By means of DFT, we show that the homogeneous intermixing of the two FMs and the artificial structural phase, where the FeCo layer is stretched to the $\operatorname{Ir}(111)$ lattice constant, leads to a narrowing and to an enhanced spin splitting of the $3 d$ states with respect to pure bulk systems. From an experimental point of view, we have induced such narrowing and enhanced spin splitting within a homogeneous $2 \mathrm{D}$ FeCo system. Therefore, our study provides an artificial 2D FeCo system protected from contaminants through the Gr layer, inducing an enhanced magnetic response, which can be further engineered for integration in magnetic devices.

See the supplementary material for selected core level measurements; additional calculations of projected DOS with different values of the Hubbard U parameter; and projected DOS for $10 \times 10 \mathrm{Gr} /$ $\mathrm{FeCo} / \mathrm{Ir}(111), 10 \times 10 \mathrm{Gr} / \mathrm{Fe} / \mathrm{Ir}(111)$, and $10 \times 10 \mathrm{Gr} / \mathrm{Co} / \mathrm{Ir}(111)$ and DOS for the corresponding FM bulk systems.

This work was partially supported by the MaX - MAterials design at the eXascale-Centre of Excellence, funded by the European Union program H2020-INFRAEDI-2018-1 (Grant No. 824143), by PRIN FERMAT (No. 2017KFY7XF) from Italian Ministry MIUR, and by Sapienza Ateneo funds. Computational time on the Marconi100 machine at CINECA was provided by the Italian ISCRA program. This work was partly performed in the framework of the nanoscience foundry and fine analysis (NFFAMIUR Italy Progetti Internazionali) facility. The authors thank A. Vegliante for his contribution to the experiment.

\section{DATA AVAILABILITY}

The data that support the findings of this study are available from the corresponding author upon reasonable request.

\section{REFERENCES}

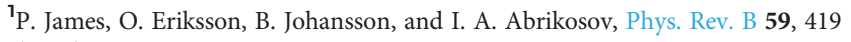
(1999).

${ }^{2}$ F. Luo, X. L. Fu, A. Winkelmann, and M. Przybylski, Appl. Phys. Lett. 91, 262512 (2007).

${ }^{3}$ M. Getzlaff, J. Bansmann, F. Bulut, R. Gebhardt, A. Kleibert, and K. MeiwesBroer, Appl. Phys. A 82, 95 (2006).

${ }^{4}$ G. Moulas, A. Lehnert, S. Rusponi, J. Zabloudil, C. Etz, S. Ouazi, M. Etzkorn, P. Bencok, P. Gambardella, P. Weinberger, and H. Brune, Phys. Rev. B 78, 214424 (2008).

${ }^{5}$ P. Gambardella, S. Rusponi, M. Veronese, S. S. Dhesi, C. Grazioli, A. Dallmeyer, I. Cabria, R. Zeller, P. H. Dederichs, K. Kern, C. Carbone, and H. Brune, Science 300, 1130 (2003).

${ }^{6}$ B. Nonas, I. Cabria, R. Zeller, P. H. Dederichs, T. Huhne, and H. Ebert, Phys. Rev. Lett. 86, 2146 (2001).

${ }^{7}$ P. Gambardella, A. Dallmeyer, K. Maiti, M. C. Malagoli, W. Eberhardt, K. Kern, and C. Carbone, Nature 416, 301 (2002).

${ }^{8}$ G. Avvisati, P. Gargiani, D. Lizzit, M. Valvidares, P. Lacovig, C. Petrillo, F. Sacchetti, and M. G. Betti, Appl. Surf. Sci. 527, 146599 (2020).

${ }^{9}$ N. Sethulakshmi, A. Mishra, P. Ajayan, Y. Kawazoe, A. K. Roy, A. K. Singh, and C. S. Tiwary, Mater. Today 27, 107 (2019).

${ }^{10}$ B. D. Terris and T. Thomson, J. Phys. D: Appl. Phys. 38, R199 (2005). 
${ }^{n_{S}}$ S. Bhatti, R. Sbiaa, A. Hirohata, H. Ohno, S. Fukami, and S. N. Piramanayagam, Mater. Today 20, 530 (2017).

${ }^{12}$ D. I. Bardos, J. Appl. Phys. 40, 1371 (1969).

${ }^{13}$ E. D. Fabrizio, G. Mazzone, C. Petrillo, and F. Sacchetti, Phys. Rev. B 40, 9502 (1989).

${ }^{14}$ C. Paduani and J. C. Krause, J. Appl. Phys. 86, 578 (1999).

${ }^{15} \mathrm{~W}$. Pepperhoff and M. Acet, Constitution and Magnetism of Iron and Its Alloys (Springer-Verlag, Berlin/Heidelberg, 2001).

${ }^{16}$ R. S. Sundar and S. C. Deevi, Int. Mater. Rev. 50, 157 (2005).

${ }^{17}$ T. Hasegawa, S. Kanatani, M. Kazaana, K. Takahashi, K. Kumagai, M. Hirao, and S. Ishio, Sci. Rep. 7, 13215 (2017).

${ }^{18}$ M. Einax, W. Dieterich, and P. Maass, Rev. Mod. Phys. 85, 921 (2013).

${ }^{19}$ M. Batzill, Surf. Sci. Rep. 67, 83 (2012).

${ }^{20}$ M. Weser, E. Voloshina, K. Horn, and Y. Dedkov, Phys. Chem. Chem. Phys. 13, 7534 (2011).

${ }^{21}$ Y. Dedkov and E. Voloshina, J. Phys.: Condens. Matter 27, 303002 (2015).

${ }^{22}$ R. Decker, J. Brede, N. Atodiresei, V. Caciuc, S. Blügel, and R. Wiesendanger, Phys. Rev. B 87, 041403 (2013).

${ }^{23}$ D. Pacilè, S. Lisi, I. D. Bernardo, M. Papagno, L. Ferrari, M. Pisarra, M. Caputo, S. K. Mahatha, P. M. Sheverdyaeva, P. Moras, P. Lacovig, S. Lizzit, A. Baraldi, M. G. Betti, and C. Carbone, Phys. Rev. B 90, 195446 (2014).

${ }^{24}$ D. Pacilè, P. Leicht, M. Papagno, P. Sheverdyaeva, P. Moras, C. Carbone, K. Krausert, L. Zielke, M. Fonin, Y. Dedkov, F. Mittendorfer, J. Doppler, A. Garhofer, and J. Redinger, Phys. Rev. B 87, 035420 (2013).

${ }^{25}$ H. Vita, S. Böttcher, P. Leicht, K. Horn, A. B. Shick, and F. Máca, Phys. Rev. B 90, 165432 (2014)

${ }^{26}$ Y. Dedkov and E. Voloshina, J. Electron. Spectrosc. Relat. Phenom. 219, 77 (2017).

${ }^{27}$ C. Cardoso, G. Avvisati, P. Gargiani, M. Sbroscia, M. S. Jagadeesh, C. Mariani, D. A. Leon, D. Varsano, A. Ferretti, and M. G. Betti, Phys. Rev. Mater. 5, 014405 (2021).

${ }^{28}$ G. Avvisati, S. Lisi, P. Gargiani, A. D. Pia, O. De Luca, D. Pacilè, C. Cardoso, D. Varsano, D. Prezzi, A. Ferretti, and M. G. Betti, J. Phys. Chem. C 121, 1639 (2017).

${ }^{29}$ M. Yang, Y. Liu, T. Fan, and D. Zhang, Prog. Mater. Sci. 110, 100652 (2020).

${ }^{30}$ R. S. Weatherup, L. D'Arsié, A. Cabrero-Vilatela, S. Caneva, R. Blume, J. Robertson, R. Schloegl, and S. Hofmann, J. Am. Chem. Soc. 137, 14358 (2015).

${ }^{31}$ M. Bazarnik, R. Decker, J. Brede, and R. Wiesendanger, Surf. Sci. 639, 70 (2015).

${ }^{32}$ E. Voloshina, B. Paulus, and Y. Dedkov, J. Phys. Chem. Lett. 12, 19 (2021).
${ }^{33}$ P. Giannozzi, S. Baroni, N. Bonini, M. Calandra, R. Car, C. Cavazzoni, D. Ceresoli, G. L. Chiarotti, M. Cococcioni, I. Dabo et al., J. Phys.: Condens. Matter 21, 395502 (2009).

${ }^{34}$ P. Giannozzi, O. Andreussi, T. Brumme, O. Bunau, M. Buongiorno Nardelli, M. Calandra, R. Car, C. Cavazzoni, D. Ceresoli, M. Cococcioni, N. Colonna, I. Carnimeo, A. Dal Corso, S. de Gironcoli, P. Delugas, R. A. DiStasio, Jr., A. Ferretti, A. Floris, G. Fratesi, G. Fugallo, R. Gebauer, U. Gerstmann, F. Giustino, T. Gorni, J. Jia, M. Kawamura, H.-Y. Ko, A. Kokalj, E. Küçükbenli, M. Lazzeri, M. Marsili, N. Marzari, F. Mauri, N. L. Nguyen, H.-V. Nguyen, A. O. de-la Roza, L. Paulatto, S. Poncé, D. Rocca, R. Sabatini, B. Santra, M. Schlipf, A. P. Seitsonen, A. Smogunov, I. Timrov, T. Thonhauser, P. Umari, N. Vast, X. Wu, and S. Baroni, J. Phys.: Condens. Matter 29, 465901 (2017).

${ }^{35}$ J. P. Perdew and A. Zunger, Phys. Rev. B 23, 5048 (1981).

${ }^{36}$ C. J. Cramer and D. G. Truhlar, Phys. Chem. Chem. Phys. 11, 10757 (2009).

${ }^{37}$ M. Cococcioni and S. De Gironcoli, Phys. Rev. B 71, 035105 (2005).

${ }^{38}$ G. Avvisati, C. Cardoso, D. Varsano, A. Ferretti, P. Gargiani, and M. G. Betti, Nano Lett. 18, 2268 (2018).

${ }^{39}$ A. Calloni, G. Bussetti, G. Avvisati, M. S. Jagadeesh, D. Pacil, A. Ferretti, D. Varsano, C. Cardoso, L. Du, F. Ciccacci, and M. G. Betti, J. Chem. Phys. 153, 214703 (2020).

${ }^{40}$ V. Popescu and A. Zunger, Phys. Rev. B 85, 085201 (2012).

${ }^{41} \mathrm{P}$. Bonfà, https://bitbucket.org/bonfus/unfold-x for the sources of the unfold-x code by P. Bonfa.

${ }^{42}$ R. Decker, M. Bazarnik, N. Atodiresei, V. Caciuc, S. Blügel, and R. Wiesendanger, J. Phys.: Condens. Mater. 26, 394004 (2014).

${ }^{43}$ A. Varykhalov, D. Marchenko, J. Sánchez-Barriga, M. R. Scholz, B. Verberck, B. Trauzettel, T. O. Wehling, C. Carbone, and O. Rader, Phys. Rev. X 2, 041017 (2012).

${ }^{44}$ J. Brede, J. Sławińska, M. Abadia, C. Rogero, J. E. Ortega, I. Piquero-Zulaica, J. Lobo-Checa, A. Arnau, and J. I. Cerdá, 2D Mater. 4, 015016 (2016).

${ }^{45}$ D. Usachov, A. Fedorov, M. M. Otrokov, A. Chikina, O. Vilkov, A. Petukhov, A. G. Rybkin, Y. M. Koroteev, E. V. Chulkov, V. K. Adamchuk, A. Grüneis, C. Laubschat, and D. V. Vyalikh, Nano Lett. 15, 2396 (2015).

${ }^{46}$ L. Massimi, S. Lisi, D. Pacilè, C. Mariani, and M. G. Betti, Beilstein J. Nanotechnol, 5, 308 (2014).

${ }^{47}$ R. H. Victora, L. M. Falicov, and S. Ishida, Phys. Rev. B 30, 3896 (1984).

${ }^{48}$ S. Pizzini, A. Fontaine, E. Dartyge, C. Giorgetti, F. Baudelet, J. P. Kappler, P. Boher, and F. Giron, Phys. Rev. B 50, 3779 (1994). 